\title{
A Study of Improving Fairness among Flows in Ad hoc Networks
}

\author{
Chisa Takano \\ Kenji Ishida \\ Masaki Aida \\ Graduate School of Information Sciences, Graduate School of Information Sciences, Graduate School of System Design, \\ Hiroshima City University, \\ 3-4-1 Ozuka-Higashi, Asa-Minami-Ku, \\ Hiroshima, 731-3194 Japan \\ Email: takano@hiroshima-cu.ac.jp \\ Hiroshima City University, \\ 3-4-1 Ozuka-Higashi, Asa-Minami-Ku, \\ Hiroshima, 731-3194 Japan \\ Email: ishida@hiroshima-cu.ac.jp \\ Tokyo Metropolitan University, \\ 6-6 Asahigaoka, Hino-shi, \\ Tokyo 191-0065 Japan \\ Email: maida@sd.tmu.ac.jp
}

\begin{abstract}
By development and the spread of a wireless technology, the mobile ad hoc network (Manet) that can be easily constructed only with mobile nodes is getting much attention. However, it is difficult to control the performance of the entire network appropriately, as a network consists of individual communication nodes operating autonomously and there is no device that controls the entire network. Recently, a large amount of research have investigated the performance of TCP in Manet, and noted the problem of leading to a larger degree of unfairness. We have proposed diffusion-type flow control (DFC) as a solution for the extremely time-sensitive flow control required for highspeed "wired" networks. In this paper, we apply the approach of DFC to Manet, in which the exchange of information across the entire network is limited, and show a wireless traffic control scheme improving the fairness among TCP flows.
\end{abstract}

\section{INTRODUCTION}

While TCP is a de facto standard protocol of the Internet that guarantees reliable delivery of data packets in wired network, TCP is demanded in mobile ad hoc networks (Manets)[1], [2], recently. Manet can construct a selfconfiguring network of mobile systems connected by wireless links without any communication infrastructure, and can offer a flexible communication environment that is robust against disasters and changes in event sites. However, there is no one specific device responsible for managing Manet. In Manet, information exchange is fundamentally and structurally limited, and each node needs to execute traffic control, path control, and network resource management on the basis only of local information.

Many techniques for Manet have been introduced, particularly with regard to the control of route selection [3], [4]. Routing algorithms that take account of security, power consumption, and Quality of Service (QoS) have been proposed [5], [6]. In addition to routing algorithms, scheduling algorithms aimed at improving the efficiency of networks offering controlled QoS have been described [7], [8].

Wireless networks, however, face not only the problem of routing but also the presence of flow control; Many studies have noted that Manet performance is severely degraded when TCP is used [9], [10], [11], [12], [13]. TCP performs congestion control on an end-to-end basis and so its response to packet loss is rather slow. To compensate this response delay, it dramatically cuts the throughput. In addition, significant TCP unfairness has been reported when TCP is used on Manet. The proposed control mechanism overcomes this weakness of TCP.

Existing autonomous distributed control schemes focus on optimizing network efficiency, i.e. optimum traffic control management and network resource utilization (battery life etc.), only in limited regions and fail to address the entire network. We have proposed the use of diffusion-type flow control (DFC) to solve similar problems in high-speed fixed networks [14], [15], [16], in which each node acts autonomously on the basis of just local information; the cumulative effect of the local decisions made at each node leads to high performance of the network as a whole. In addition, we have investigated the impact of DFC on the performance of highspeed networks. DFC uses only the information it is aware to determine the transmission rate. DFC avoids packet loss due to buffer overflow by ensuring that the density of packets stored in each node is not unevenly distributed to certain nodes, but is adequately diffused over the whole network. The diffusion effect associated with DFC leads to steady data transmission even in high-speed networks. Moreover, the cost of the network can be reduced because the capacity of the storage buffers in the nodes can be reduced.

This paper applies the framework of DFC to Manet, and investigates the feasibility of this in environments where information exchange is fundamentally and structurally limited. In the DFC implementations described in the past, each node had to know the propagation delay on the links to adjacent nodes. However, in Manet it is difficult to know the propagation delay in real time because the distance to the other hosts is continually changing. Therefore, it is impractical to demand knowledge of the link delay to adjacent hosts. In this paper, we extend DFC so that it can be applied in a wireless environment and introduce a control method that doesn't depend on link length. Then, we show that a combination of DFC and TCP achieves higher network performance than TCP in Manet.

The remainder of this paper is organized as follows. Sec. II describes the framework of DFC and the basic operation of DFC for wired networks, and then proposes a DFC variant in which the control timing interval, that is the time interval at 

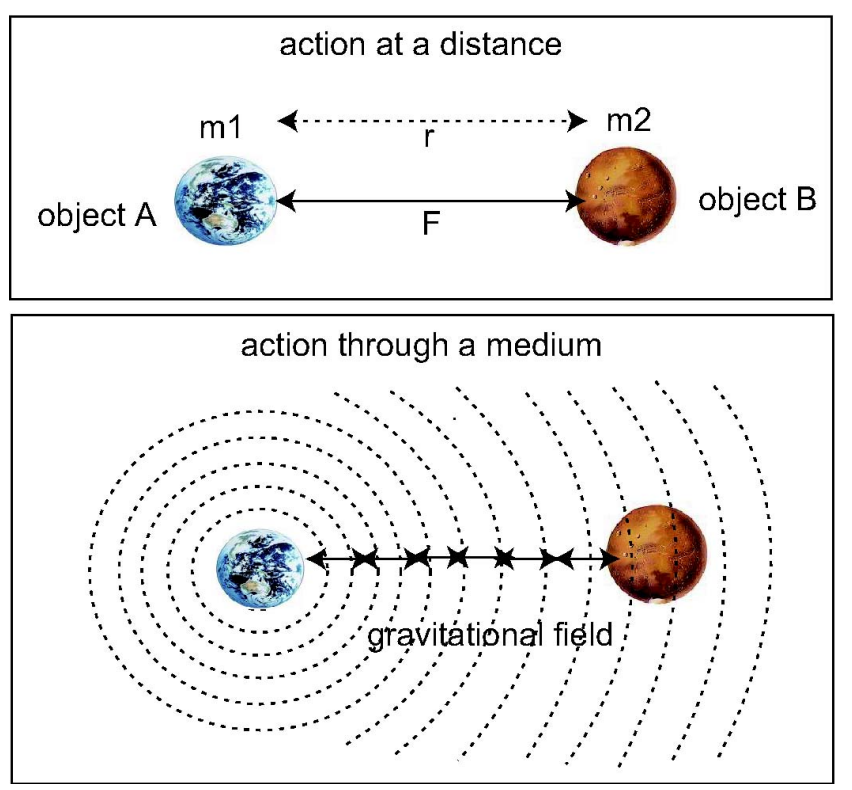

Fig. 1. Action at a distance and action through a medium.

which flow control is applied, is independent of the link length. Conditions for simulating this new technique and simulation results are described in Sec. III. Finally, Conclusions are drawn in Sec. IV.

\section{Diffusion-Type Flow CONTROL MeChanism}

In this section, we describe the framework of DFC as applied to high-speed wired networks.

\section{A. DFC applying Action Through Medium}

When thinking about the interaction between two objects which are separated in space, there are two theories depending on the action method; "action at a distance" and "action through a medium"(Fig. 1). "Action at a distance" is the direct interaction of two objects that are not linked by any field or medium. For example, Newton's law of universal gravitation is on the basis of "action at a distance", and the force $F$ of gravity acting between object $A$ and object $B$ is directly proportional to the mass $m_{1}$ and $m_{2}$ of both interacting objects, and inversely proportional to the square of the distance $r$ between object $\mathrm{A}$ and $\mathrm{B}$ as follows:

$$
F=G \frac{m_{1} m_{2}}{r^{2}}
$$

where $G$ is the gravitational constant. The function $F$ is decided by the mass of objects and the distance, that is to say that it is necessary to know the values of the mass and the distance. From the point of view of a network control, this is same as the framework of the centralized control to require the collection of global information about the network state.

In the case of "action through a medium", on the other hand, variation of the physical value at a point of space is transmitted to the adjacent point via the medium of the gravitational

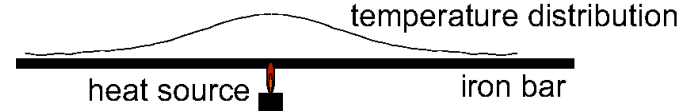

Fig. 2. Example of thermal diffusion.

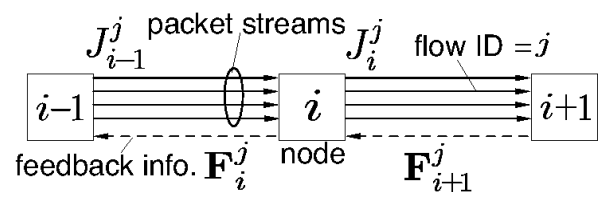

Fig. 3. Node interaction in our flow control model.

field at the limited speed. A temporal variation in such a gravitational field can be described as a partial differential equation.

We use the following example to elucidate "action through a medium". When we heat one end of an iron bar, the temperature distribution follows a normal distribution and heat spreads through the whole bar by diffusion (Fig. 2). In this process, the action in a minute segment of the iron bar is very simple: heat flows from the hotter side towards the cooler side. The rate of heat flow is proportional to the temperature gradient. Although each segment acts autonomously, based on its local information, the temperature distribution of the whole iron bar exhibits orderly behavior.

In DFC, each node controls its local packet flow to an adjacent node so that it is proportional to the difference between the number of packets in that node and the number in the adjacent node. The overall effect is that the distribution of the total number of packets in each node in the network becomes uniform over time.

\section{B. Behavior of Diffusion-Type Flow Control Mechanism}

Figure 3 shows the interactions between nodes (routers) in DFC, using a network model with a simple 1-dimensional configuration. In DFC, node $i(i=1,2, \ldots)$ transfers packets to node $i+1$, and node $i+1$ sends feedback information $\mathbf{F}_{i+1}$ to node $i$. When node $i$ receives feedback information from downstream node $i+1$, it determines the transmission rate for packets to the downstream node $i+1$ using the received feedback information, and it adjusts its transmission rate towards the downstream node $i+1$ accordingly. Let us assume that there are $M_{i}$ flows sharing the link between node $i$ and node $i+1$, and they are identified by $j\left(j=1,2, \ldots, M_{i}\right)$. Each node $i$ autonomously determines the transmission rate $J_{i}^{j}$ for flow $j$ on the basis of the feedback information obtained from the downstream node $i+1$ and its own information. The transmission of packets and feedback information both experience the same propagation delay.

The transmission rate $J_{i}^{j}(t)$ for flow $j$ of node $i$ at time $t$ is determined by

$$
\begin{aligned}
J_{i}^{j}(t) & =\max \left(0, \min \left(L_{i}^{j}(t), \tilde{J}_{i}^{j}(t)\right)\right), \\
\tilde{J}_{i}^{j}(t) & =r_{i}^{j}\left(t-d_{i}\right)-D_{i}\left(n_{i+1}^{j}\left(t-d_{i}\right)-n_{i}^{j}(t)\right),
\end{aligned}
$$


where $L_{i}^{j}(t)$ denotes the available bandwidth for flow $j$ of the link from node $i$ to node $i+1$ at time $t, n_{i}^{j}(t)$ denotes the number of packets belonging to flow $j$ in node $i$ at time $t, r_{i}^{j}\left(t-d_{i}\right)$ is the required rate for flow $j$ derived from the feedback information from the downstream node $i+1$ (hereafter called the "notified rate"), and $d_{i}$ denotes the propagation delay between nodes $i$ and $i+1 . D_{i}$ is a parameter used by DFC.

Let the bandwidth of the link from node $i$ to node $i+1$ be $B_{i}$, and $L_{i}^{j}(t)$, the available bandwidth for flow $j$, is derived by assuming that the bandwidth $B_{i}$ is shared by the different flows according to weight $W_{i}^{j}(t)$, that is,

$$
\begin{aligned}
K_{i}^{j}(t) & =\frac{B_{i}}{\sum_{k=1}^{M_{i}} 1_{\{k=\text { active }\}}}-D_{i}\left(n_{i+1}^{j}\left(t-d_{i}\right)-n_{i}^{j}(t)\right) \\
W_{i}^{j}(t) & =\frac{K_{i}^{j}(t)}{\sum_{k=1}^{M_{i}} 1_{\{k=\text { active }\}} \times K_{i}^{k}(t)} \\
L_{i}^{j}(t) & =W_{i}^{j}(t) \times B_{i}
\end{aligned}
$$

where $1_{\{k=\text { active }\}}$ is the indicator function; it is equal to 1 if flow $k$ is active at time $t$, otherwise 0 . This rule means that a flow with the larger second term of (2) can get a larger transmission rate and can transmit a larger volume of traffic to the downstream node.

The feedback information for flow $j$ created at fixed intervals $\tau_{i}$ by node $i$ consists of three quantities, as follows:

$$
\mathbf{F}_{i}^{j}(t)=\left(r_{i-1}^{j}(t), n_{i}^{j}(t), \ell_{i}^{j}(t)\right)
$$

When implementing DFC in a wired network, we assume that the control timing interval is equal to the propagation delay $\left(\tau_{i}=d_{i-1}\right) \cdot \ell_{i}^{j}(t)$ is the information defining the maximum available bandwidth. $\ell_{i}^{j}(t)$ and the notified rate $r_{i-1}^{j}(t)$ are limited by the available bandwidth $L_{i}^{j}$ as follows.

$$
\begin{aligned}
\ell_{i}^{j}(t) & =\min \left(L_{i}^{j}(t), \ell_{i+1}^{j}(t)\right), \\
r_{i-1}^{j}(t) & =\max \left(0, \min \left(L_{i}^{j}(t)\right), \tilde{J}_{i}^{j}(t)\right) .
\end{aligned}
$$

C. Parameter design independent of the link propagation delay

In some previous works, parameter $D_{i}$ was chosen to be inversely proportional to the propagation delay as follows:

$$
D_{i}=\frac{D}{d_{i}},
$$

where $D(>0)$, which is a positive constant, is the diffusion coefficient. As shown in [16], setting parameter $D_{i}$ in accordance with Eq.(9) allows the complexity of the network model to be handled, and provides high performance and stability even if the configuration of the network becomes complex.

Although we assumed $d_{i}=\tau_{i+1}$ in the previous subsection, the two parameters have different origins, and so we can regard them as different (independent) items. Therefore, we show that, for more general applicability, parameter $D_{i}$ should be

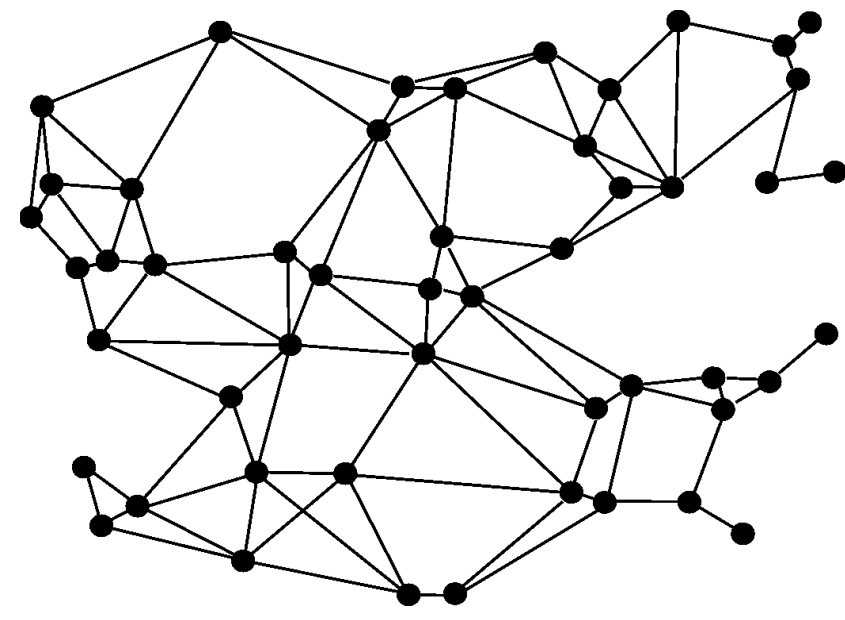

Fig. 4. Simulation model.

determined by using the control timing interval $\tau_{i}$ (not the propagation delay $d i$ ) in accordance with [17] as follows.

$$
D_{i}=\frac{D}{\tau_{i+1}}
$$

where the interval of DFC's actions $\tau_{i}$ is unchanged and $\tau_{i}$ is independent of $d_{i}$.

To appropriately associate the number of packets $n_{i}^{j}(t)$ in a discrete space with the packet density $n^{j}(x, t)$ in a continuous space, Eq.(2) needs to be modified for the continuous case, which is achieved by the following equation .

$$
\tilde{J}^{j}(x, t)=r^{j}(x, t)-\kappa \frac{\partial n^{j}(x, t)}{\partial x},
$$

where $\kappa$ is the diffusion coefficient of the physical diffusion phenomenon (in the continuous space). We have Eq. (10) by the relation $D=\kappa \Delta t /(\Delta x)^{2}$ which is already known from [16]. It is obvious from a consideration of the relationship between $\kappa$ and $D$. To guarantee the diffusion effect for any conditions, it is necessary to adopt the value of $D$ in the range as follows[16]:

$$
0<D<\frac{1}{2} .
$$

\section{SIMULATIONS}

In this section, we apply the DFC approach to a Manet in which the hosts are moving, and show that overall Manet performance can be optimized even though each node is operating autonomously and processing only local information. We extend the simulation tool ns2 capability with the function of DFC to investigate the performance of DFC when coexisting with TCP.

\section{A. Simulation Model}

Figure 4 shows the network model used in the simulations, which includes 50 mobile hosts. Black circles denotes the source and destination nodes in Manet, and black lines show 


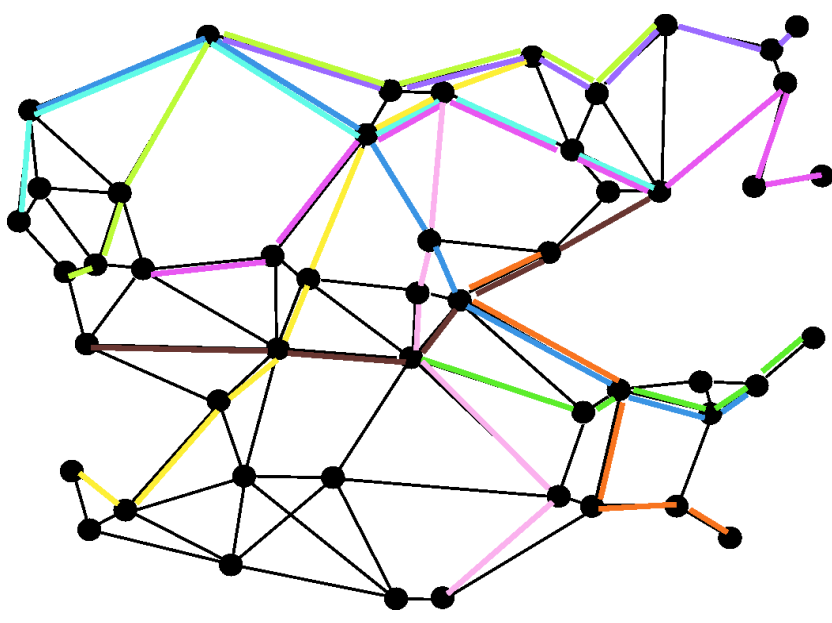

Fig. 5. Route of Flows.

the wireless links between nodes. The size of the area is about $1.2 \mathrm{~km} \times 0.9 \mathrm{~km}$, and each node is arranged at random as an initial state. The migration speed of each host is $10 \mathrm{~km} /$ hour and the migration angle is a random value between 0 and $2 \pi$. $D$ is equal to 0.4 . Each packet has a fixed length of 1,500 Bytes and the link bandwidth is 4,400 packets/s which corresponds to a link bandwidth of $54 \mathrm{Mbps}$.

The simulations described here assume a non-uniform network configuration, that is, links have different lengths. The propagation delay of each link is determined by dividing link length by the speed of light.

The basic simulation scenario is as follows. There are ten flows; The first flow starts at simulation time $t=0 \mathrm{sec}$ and the others are added to Manet every five seconds. The simulation end time is $50 \mathrm{sec}$. All flows are greedy, that is, the rate of each flow is as large as possible. The maximum buffer capacity for each flow at each host is 1,000 packets.

In DFC, all hosts use the same the control timing interval 50 msec. Parameter $D_{i}$ is set by Eq.(10). The maximum TCP window size and the initial TCP window size of both flows are 10,000 ; this value was chosen as it is sufficiently larger than the bandwidth-delay product of RTT. The TCP implementation used in the model is TCP Reno.

\section{B. Evaluation of the fairness among flows}

We investigate the characteristics of throughput for each flow when (a) TCP flow control without DFC and (b) TCP with DFC is used in Manet. In this paper, we use the shortest hop algorithm which the route construction schemes of the existing routing algorithms usually use in Manet. The route of ten flow is shown in Fig. 5, and the maximum number of the flow in one link is three.

First, the result when only the TCP is used in Manet is shown in Fig. 6. The horizontal axes denote the simulation time and the vertical axes denote the throughput (Mbit/sec) for each flow. The ten lines in this figure denote the results for the ten flows. Throughput is calculated as the amount (Mbit)

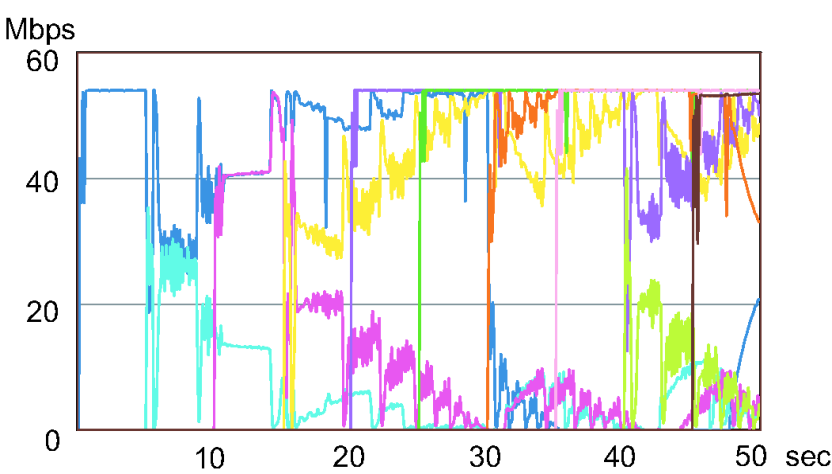

Fig. 6. Throughput in the case of TCP without DFC.

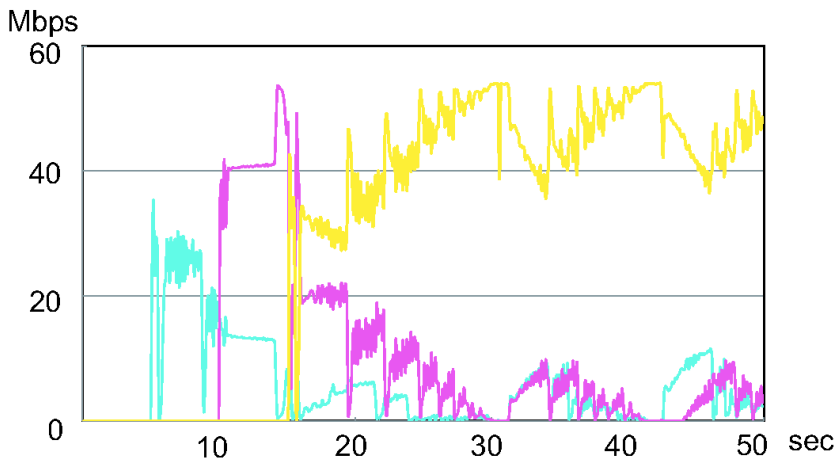

Fig. 7. Throughput of flow 2, 3 and 4 (TCP without DFC).

of the packet that the destination node received per second. The calculation granularity is assumed to be $0.1 \mathrm{sec}$.

In Fig. 6, the throughput of TCP without DFC is unstable for each flow, and some flows have high throughput, but the others do not. Figure 7 shows the results of flow 2 (blue), 3 (red), and 4 (orange) extracted from Fig. 6. These flows share some link at simulation time $15 \mathrm{sec}$. Because three flows share the link having the link bandwidth of $54 \mathrm{Mbps}$, the bandwidth which one flow can use becomes $18 \mathrm{Mbps}$ if these flows are fair. But we see from Fig.7 that the throughput of flow 4 is higher than flow 2 and 3, while the throughput of flow 2 and flow 3 falls to zero. This is because the new traffic entering Manet leads to packet losses of flow 2 and 3 and TCP reduces its window size for flow 2 and 3. Such unfairness is caused because flow 4 can use the remaining bandwidth that flow 2 and 3 do not use.

Figures 8 and 9 show the results obtained from TCP with DFC. Simulation condition is same as Fig. 6 and Fig.7. We can see from these figures that the throughput of each flow is steady and the value of throughput for each flow reaches the ideal value $18 \mathrm{Mbps}$. Because the number of packets stored at nodes becomes uniform over the network through the diffusion effect of DFC and packet losses can occur not, the throughput of each flow are fair.

Next, we change the value of the link cost by way of 


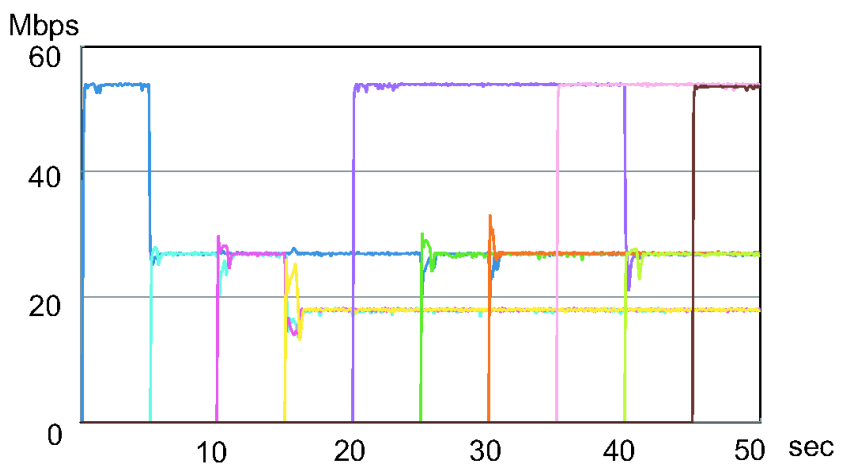

Fig. 8. Throughput in the case of TCP with DFC.

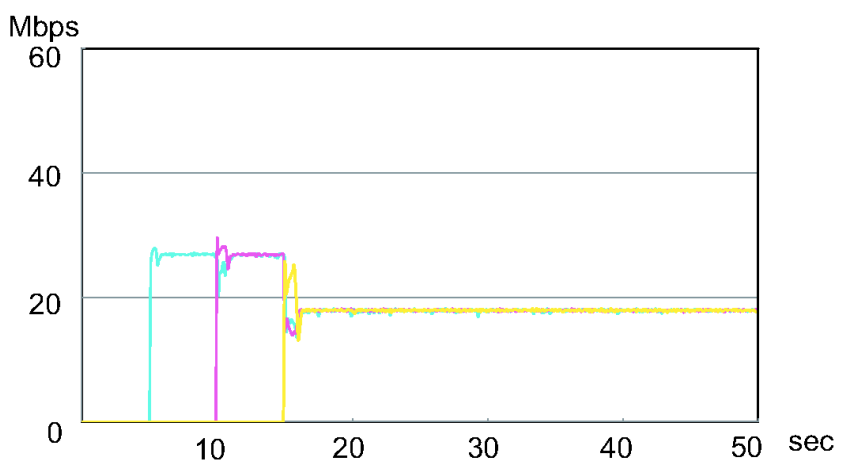

Fig. 9. Throughput of flow 2, 3 and 4 (TCP with DFC).

experiment, and we consider the situation in which more flows share the link. The link cost is set to uniform random number between $(8,12)$. The route of flows is calculated by using the shortest hop algorithm. Under this condition, the maximum number of the flow shared in one link is four (Fig. 10).

The result when only the TCP is used in Manet is shown in Fig. 11. The horizontal axes denote the simulation time and the vertical axes denote the throughput (Mbit/sec) for each flow. In Fig. 11, the throughput of TCP without DFC is unstable for each flow, the same as Fig. 6. Here, we focus on four flows (flow 3, 6, 9 and 10) as shown in Fig. 11, where the value the vertical axes is between $45 \mathrm{sec}$ and $50 \mathrm{sec}$. (Flow 10 starts at $45 \mathrm{sec}$.) We see from this figure that unfairness is caused among four flows.

On the other hand, Fig. 13 and 14 show results when TCP with DFC is used. We can see from these figures that the throughput of each flow is steady and the value of throughput for each flow reaches the ideal value $13.5 \mathrm{Mbps}$ (54 Mbps/4 flows).

\section{CONCLUSION}

We have proposed DFC to overcome the difficulty of controlling high-speed networks. In this paper, we used DFC to control Manet, and verified that DFC works well even when the exchange of information across the entire network is limited. We investigated the characteristics of throughput

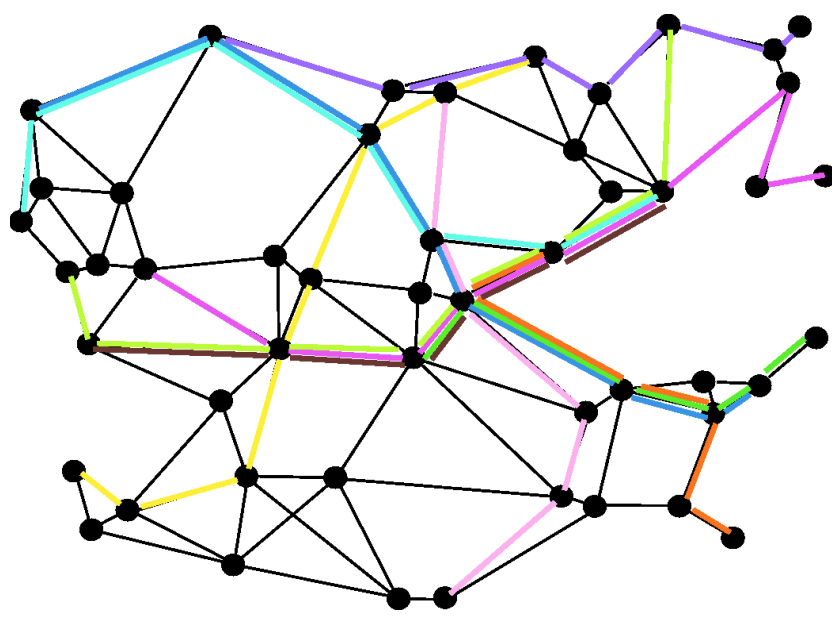

Fig. 10. Route of Flows. (random link cost)

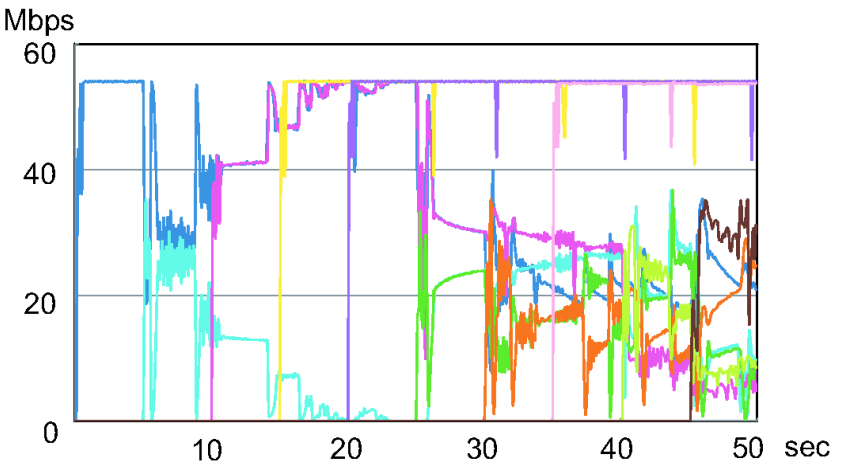

Fig. 11. Throughput in the case of TCP without DFC. (random link cost model)

for each flow when TCP flow control without DFC or TCP with DFC is used in Manet. The simulation results showed that unfairness is caused in the case of TCP without DFC. This is because the congestion occurring in Manet leads to the packet losses of some flow and TCP reduces its window size. In addition, the other flows sharing the link with this flow can use the remaining bandwidth.

Moreover, we investigated the performances of TCP with DFC and we found that the throughput of each flow is steady and the value of throughput for each flow reaches the ideal value. These results indicate that the desirable characteristics of DFC, including the cooperative behavior of nodes to avoid packet loss and the ability to recover rapidly from congestion, are also effective for a TCP controlled flow in Manet.

\section{ACKNOWLEDGMENT}

This work was partly supported by Grant-in-Aid for Young Scientists (B) (21700086) from the Japan Society for the Promotion of Science and the Ministry of Internal Affairs and Communications of Japan under Grant-in-Aid for Strategic Information and Communications R\&D Promotion Programme 


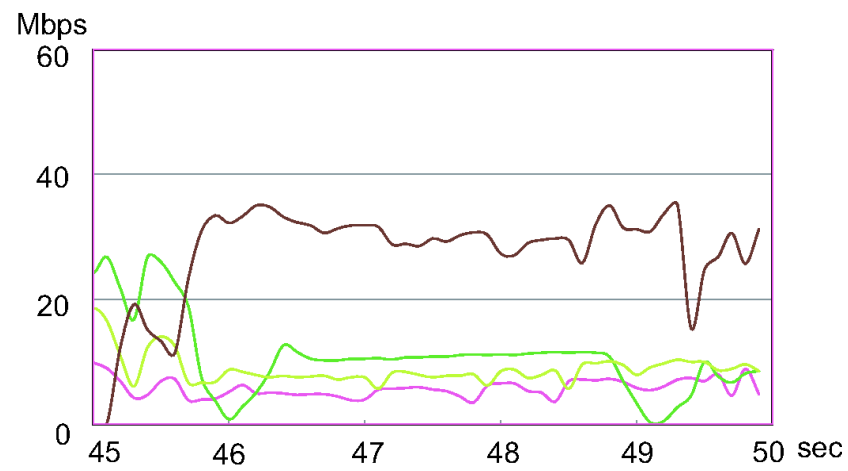

Fig. 12. Throughput of flow 3, 6, 9 and 10 (TCP without DFC). (random link cost model)

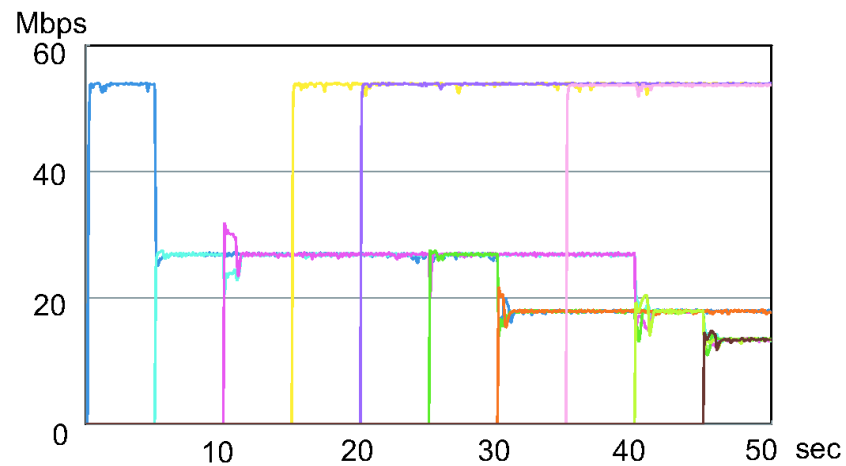

Fig. 13. Throughput in the case of TCP with DFC. (random link cost model)

(SCOPE).

\section{REFERENCES}

[1] http://www.ietf.org/html.charters/manet-charter.html

[2] E. Royer and C.-K. Toh, "A review of current routing protocols for ad hoc wireless networks," IEEE Personal Communications, pp. 46-55, April 1999.

[3] E. Perkins, M. Belding-Royer and R. Das, "Ad hoc on-demand distance vector (AODV) routing," Request for Comments 3561, July 2003.

[4] T. Clausen and P. Jacquet, "Optimized link state routing protocol (OLSR),” Request for Comments 3626, October 2003.

[5] Y. Hu, A. Perrig, and B. Johnson, "Ariadne: A Secure on-demand routing protocol for ad hoc networks," in Proceedings of ACM MobiCom 2002, Sept. 2002.

[6] Y. Xu, J. Heidemann and D. Estrin, "Geography-informed energy conservation for ad hoc routing," in Proceedings of ACM Mobile Computing and Networking 2001, July 2001.

[7] X. Lin and N. B. Shro , "Joint rate control and scheduling in multihop wireless networks," in Proceedings of IEEE Conference on Decision and Control, Bahamas, 2004.

[8] L. Bui, A. Eryilmaz and R. Srikant, "Joint Asynchronous Congestion Control and Distributed Scheduling for Multi-Hop Wireless Networks," in Proceedings of IEEE INFOCOM 2006, Apr. 2006.

[9] H. Balakrishnan, V. Padmanabhan, S. Seshan, and R. H. Katz. , "A Comparison of Mechanisms for Improving TCP Performance over Wireless Links," IEEE/ACM Transactions on Networking, Dec. 1997.

[10] M. Gerla, R. Bagrodia, L. Zhang, K. Tang, and L. Wang, "TCP over Wireless Multihop Protocols: Simulation and Experiments," IEEE ICC 1999.

[11] G. Holland and N. Vaidya, "Analysis of TCP Performance over Mobile Ad Hoc Networks,” ACM MOBICOM 1999.

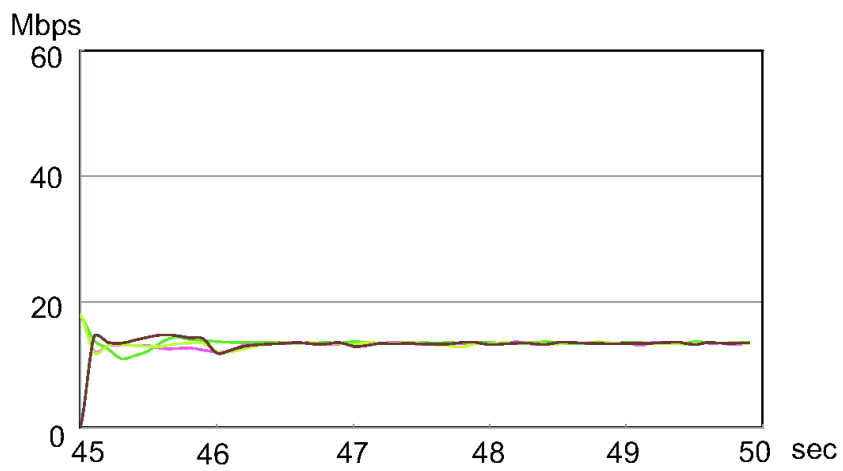

Fig. 14. Throughput of flow 3, 6, 9 and 10 (TCP with DFC). (random link cost model)

[12] Zhenghua Fu, Petros Zerfos, Haiyun Luo, Songwu Lu, Lixia Zhang, and Mario Gerla, "The impact of multihop wireless channel on TCP throughput and loss," Proc. of 22th Annual Joint Conference of the IEEE Computer and Communications Societies, pp. 1744-1753, IEEE, San Francisco, USA, Mar. 2003.

[13] Li-Ping Tung, Wei-Kuan Shih, Te-Chung Cho, Yeali S. Sun, and Meng Chang Chen, "TCP Throughput Enhancement over Wireless Mesh Networks," IEEE Communications Magazine, vol. 45, no. 11, pp. 64-70, Nov. 2007.

[14] C. Takano and M. Aida, "Diffusion-type autonomous decentralized flow control for end-to-end flow in high-speed networks," IEICE Transactions on Communications, vol.E88-B, no.4, pp.1559-1567, 2005.

[15] C. Takano and M. Aida, "Diffusion-type autonomous decentralized flow control for multiple flows," IEICE Transactions on Communications, vol. E90-B, no. 1, pp. 21-30, January 2007.

[16] C. Takano, K. Sugiyama and M. Aida, "Parameter Design for DiffusionType Autonomous Decentralized Flow Control,' IEICE Transactions on Communications, vol. E91-B, no. 9, pp. 2828-2837, September 2008.

[17] C. Takano and M. Aida, "Autonomous decentralized flow control allowing control timing independent of link length," IEEE ISADS 2009 Workshops (AHSP 2009), Athens, Greece, March 23-25, 2009. 